\title{
Téoros
}

Revue de recherche en tourisme

\section{Patrimoine mondial}

Les enjeux locaux

\section{Lucie K. Morisset et Mathieu Dormaels}

Volume 30, numéro 2, 2011

Tourisme et patrimoine mondial

URI : https://id.erudit.org/iderudit/1012236ar

DOI : https://doi.org/10.7202/1012236ar

Aller au sommaire du numéro

Éditeur(s)

Université du Québec à Montréal

ISSN

0712-8657 (imprimé)

1923-2705 (numérique)

Découvrir la revue

Citer ce document

Morisset, L. K. \& Dormaels, M. (2011). Patrimoine mondial : les enjeux locaux.

Téoros, 30(2), 3-5. https://doi.org/10.7202/1012236ar

Ce document est protégé par la loi sur le droit d'auteur. L'utilisation des services d'Érudit (y compris la reproduction) est assujettie à sa politique d'utilisation que vous pouvez consulter en ligne.

https://apropos.erudit.org/fr/usagers/politique-dutilisation/
Cet article est diffusé et préservé par Érudit.

Érudit est un consortium interuniversitaire sans but lucratif composé de l’Université de Montréal, l'Université Laval et l'Université du Québec à Montréal. Il a pour mission la promotion et la valorisation de la recherche. https://www.erudit.org/fr/ 


\title{
Patrimoine mondial Les enjeux locaux
}

\author{
Lucie K. MORISSET, MSRC \\ Professeure \\ Département d'études urbaines et touristiques (ESG-UQAM) \\ morisset.lucie@uqam.ca \\ Mathieu DORMAELS \\ Chercheur associé \\ Chaire de recherche du Canada en patrimoine urbain (ESG-UQAM) \\ mat_dormaels@yahoo.com
}

L'interrelation entre le patrimoine et le tourisme, pour multiforme qu'elle puisse être historiquement et géographiquement, fait dorénavant consensus parmi les chercheurs et les praticiens. Aussi peut-on concevoir, au moins a priori, que la croissance phénoménale du tourisme international ait pour corollaire une expansion équivalente d'un patrimoine particulier qui puisse se comparer sur la scène planétaire, où l'on mesure aussi la densification des flux touristiques : c'est le patrimoine mondial. Pourrait-on même croire, compte tenu de l'inflation patrimoniale qui voit croître sans cesse le corpus des objets et des lieux dotés d'un tel qualificatif, que le patrimoine puisse catalyser un redéploiement du tourisme, tant eu égard à ses pratiques qu'à ses composantes industrielles?

En effet, malgré les crises et autres aléas des dernières années, les arrivées de touristes internationaux ont augmenté de $4 \%$ en 2011, et l'OMT s'attend à ce que leur nombre dépasse le milliard en $2012^{1}$. Que l'on accepte ou non de considérer ces chiffres comme des indicateurs efficaces du phénomène touristique, ils restent indicateurs de flux économiques importants. Leurs représentations ont, depuis une cinquantaine d'années, tendu à minimiser le poids des attraits culturels, a fortiori patrimoniaux, réduits au statut de créneau d'un marché bien plus vaste et surtout plus complexe. Pourtant, le patrimoine règne sur une part importante des produits et des destinations touristiques : quel séjour n'invite pas à visiter un village typique, quelle croisière n'enfile pas les sites du patrimoine mondial?

On touche ici au noyau des interrelations que ce dossier de Téoros voudrait aborder autour de l'une des déclinaisons les plus probantes du phénomène patrimonial dans l'actualité touristique, le patrimoine mondial. La Liste des sites inscrits au patrimoine mondial de l'UNESCO, c'est-à-dire des sites reconnus pour leur "valeur universelle exceptionnelle» en vertu de la Convention concernant la protection du patrimoine mondial, culturel et naturel (1972), semble en effet être aussi devenue un gage de qualité pour les promoteurs touristiques, telle une sorte de must see list offrant en retour aux autorités locales le sésame d'une prospérité nouvelle grâce au développement local conquis avec les retombées économiques du tourisme.

En deçà de l'effet bien réel de labellisation d'une désignation dont la dénomination institue la planète pour cadre de référence et situe conséquemment ses objets à l'apex de l'humanité, le patrimoine a de quoi séduire le tourisme. En effet, celui-là aspire à véhiculer les expressions identitaires que celui-ci prise : en tant que représentation d'une particularisation et d'une différence voulant que ce que l'on trouve ainsi ne se retrouve pas ailleurs, le patrimoine est l'inventeur de l'exotisme et du dépaysement. Pour peu que l'on renverse la lunette qu'a adoptée l'industrie touristique dans la foulée de sa professionnalisation et de son institutionnalisation, le patrimoine est une figure de proue des déterminants du tourisme, c'est-à-dire d'une industrie structurée, en deçà des services qui en densifient l'exploitation, par une ressource et sa mise en valeur. Les remonte-pentes restent généralement périphériques dans la décision d'un skieur d'aller à Chamonix ou au mont Sainte-Anne. Les efforts récents de positionnement de certains émirats arabes, pour rester dans l'univers de l'industrie, sont au demeurant révélateurs de la pérennité d'un constat : protagoniste des ressources culturelles, le patrimoine a pour qualité d'outrepasser la durée de vie de toute campagne de marketing, car son objectalité et ses imaginaires transcendent les modes et les générations, celles des touristes comme celles des promoteurs.

Dans cette perspective, la protection et la valorisation du patrimoine, telle qu'elles peuvent être comprises en transparence des intentions de la Convention concernant la protection du patrimoine mondial, culturel et naturel, deviennent des éléments fondamentaux de l'attractivité des destinations. On comprend alors l'intérêt réciproque d'une gestion conjointe des services et des ressources, ainsi que des représentations culturelles des uns et des attraits exotiques des autres, plus 
encore lorsque l'exceptionnalité, tout en attirant plus de visiteurs, rend la conservation indispensable.

Comme la montagne ou la jungle des explorateurs, c'est d'ailleurs par le tourisme que s'est avéré le patrimoine de l'Italie, de la France, puis de la Grèce, de l'Égypte par exemple, sillonnées par le Tour des Anglais; c'est ainsi qu'est né le patrimoine du Québec sur les parcours des Américains. Les études se sont d'ailleurs multipliées ces dernières années, notamment dans le champ des cultural studies, pour confirmer ce rapport d'influence entre site et sight (on nous permettra de paraphraser ici Dean MacCannell) et, surtout, pour rappeler qu'au cœur de l'expérience touristique comme de l'enthousiasme identitaire, c'est le patrimoine qui, dans le temps long et pour longtemps, définit la différence et la particularité.

On comprend donc que, si un site est «plus patrimonial» parce qu'inscrit au patrimoine mondial en vertu d'une valeur universelle, il soit d'autant plus touristique ou appelé à le devenir. Bien entendu, cette logique, qui porte aussi à un point culminant les antagonismes variés entre patrimoine et commerce, soulève un certain nombre de questions et notamment celle de la motivation à créer du patrimoine pour favoriser le tourisme, ce qui ne manque pas d'émouvoir les partisans de l'authenticité. Là encore, en effet, le patrimoine mondial est en ligne de mire, avec des statistiques d'accroissement de fréquentation tout aussi mirobolantes que l'étendue de la clientèle que son vocable planétaire convoque et avec, il faut bien le dire, ce qui pourrait apparaitre comme une forme d'opportunisme réciproque entre une instance chargée de reconnaître la valeur exceptionnelle et des acteurs soucieux, eux, de développement ou de rentabilité. La signature en 2009 d'un accord de partenariat entre le Centre du patrimoine mondial, organe chargé de l'administration de la Liste, et l'entreprise TripAdvisor incite au moins les plus cyniques à sourire, quand on constate parallèlement que la Liste en question est passée en à peine 30 ans de 12 sites à plus de 900 .

Dans la foulée, d'ailleurs, et dans la plus grande confusion, un certain nombre de "listes réduites" d'initiative privée ont vu le jour ces derniers temps, telles les New 7 Wonders qui par surcroît opposent aux mécanismes de l'UNESCO une prétention (assez peu avérée) au suffrage universel. Outre les difficultés d'administration et d'imputation de moyens financiers et humains que soulèvent simultanément la croissance exponentielle et la popularité grandissante de la Liste du patrimoine mondial, son rôle évident de marqueur de qualité touristique, pour les marchands du temple comme pour les autres, incite au questionnement et au débat.

On peut ainsi réfléchir, bien sûr, aux intérêts réels de consolider les liens entre patrimoine et tourisme, si tant est que la densité de l'expérience touristique, jaugée à l'aune mondiale, affecte, sinon toujours la véracité, au moins la préservation de ressources patrimoniales destinées à être mesurées à la même échelle. Comment faire en sorte qu'un site plus visité parce qu'il est plus important le soit moins intensément afin, comme le veut sa vocation patrimoniale, de l'être plus longtemps, jusqu'aux générations futures? Pire, l'accroissement de la mobilité et des échanges induit par le tourisme ne risque-t-il pas à terme de contaminer les patrimoines, de les dissoudre en quelque sorte dans la mondialisation?
Non plus que le patrimoine mondial soit un ignorant label, le tourisme n'est pas une simple consommation qui condamnerait ses produits à une digestion indifférente. Pour autant, le rapport (bénéfique, croyons-nous) d'influence entre tourisme et patrimoine met en jeu, lorsqu'il est posé dans un cadre de référence supranational et planétaire, un basculement d'échelles avec lequel on cesse de ressentir un certain inconfort. Bien sûr, avant d'être de valeur universelle, le site du patrimoine mondial est reconnu et promu en son territoire (dans l'immense majorité des cas) par l'État qui revendique la responsabilité de sa sélection et de sa protection. La vitrine mondiale de la Liste qui l'offre subséquemment au tourisme international ne serait donc qu'un agrandissement. Mais un agrandissement de quoi? Un patrimoine peut-il, en effet, être mondial? Un objet ou un site peut-il, aux yeux de tous autant que sous le regard de ceux qu'il représente localement, caractériser une historicité donnée, et ce, à l'échelle planétaire? Peut-on penser que l'humanité ait en commun des valeurs qui puissent investir un lieu d'un sens unanime et partageable par tout un chacun, hôte ou touriste, austral ou septentrional, occidental ou oriental, jeune ou ancien?

Ces interrogations, qui rejoignent le noyau des postcolonialstudies, ouvrent bien sûr une boîte de Pandore que nous n'entendons pas refermer. Dans le monde touristique, le patrimoine mondial soulève d'ailleurs bien d'autres enjeux qu'il est aussi possible de questionner en quête de réflexions d'une portée plus immédiate, tant que l'on accepte de considérer le tourisme mû par des ressources parmi lesquelles le patrimoine n'est pas qu'un segment de marché, mais un moteur décisif.

La question n'est ainsi plus de savoir s'il faut prendre en compte le tourisme dans la gestion du patrimoine ou s'il faut mettre en valeur le patrimoine pour les touristes, mais plutôt de se demander comment valoriser le patrimoine mondial et quelle est la réalité de situations aussi différentes que peuvent l'être les sites eux-mêmes. En effet, face à la motivation croissante et explicite des autorités locales et nationales de régénérer économiquement une région par le tourisme, plusieurs études ont été, ou sont encore, menées pour tenter de mesurer les retombées réelles de l'inscription et des différentes valorisations qui sont mises en place. Y a-t-il - et quels sont-ils - des effets sur l'économie et le développement local?

C'est à ces questionnements que répondent les textes présentés dans ce dossier. Lionel Prigent pose très directement la question des retombées de l'inscription dans une perspective économique puisqu'il constate que, si les études menées récemment portent à relativiser l'impact de l'inscription sur les économies locales, il demeure une impression croissante d'un lien entre tourisme et patrimoine mondial. Se pose alors la question du pourquoi de cette motivation qui pousse les autorités à investir dans cette reconnaissance puisque le patrimoine mondial semble plutôt être un «mirage économique». Sa réflexion sur les liens entre l'expérience touristique et la nature du patrimoine, illustrée par le cas du Mont-SaintMichel, en France, dévoile des conséquences méconnues de l'inscription de sites qui requièrent une mise en valeur et un entretien coûteux. C'est finalement à travers l'expression même de nos valeurs contemporaines qu'il propose de comprendre ce lien entre patrimoine et tourisme. 
Pour sa part, Luc Florent examine les implications de la labellisation de l'inscription sur la Liste du patrimoine mondial et de l'impact d'un tel label sur les touristes. Si le terme de «label» est en effet souvent utilisé, rares sont les études qui interrogent la pertinence de son utilisation, avant tout conditionnée dans la promotion commerciale et le marketing. Florent s'intéresse par ailleurs à la relation entre la connaissance de ce label par les touristes, son utilisation par les autorités responsables des sites et la notoriété de ceux-ci. Son analyse met en évidence des différences effectives entre les sites inscrits, qui sont déjà connus et reconnus, pour lesquels l'inscription ne paraît pas représenter un apport majeur, et ceux moins notoires pour lesquels la reconnaissance semble un outil de promotion nécessaire. Prigent et Florent ouvrent donc une perspective différente sur la relation entre tourisme et patrimoine mondial qui propose de lire les valeurs revendiquées par les communautés locales dans les choix de valorisation touristique du patrimoine et suggère que l'influence de l'inscription soit plutôt perceptible dans les décisions prises par les touristes et moins dans d'éventuelles retombées économiques.

Les trois textes qui suivent soulèvent eux aussi des questions importantes quant à la relation entre tourisme et patrimoine mondial, grâce à l'étude de cas particuliers. Maie Gérardot présente le cas de la ville de Budapest, site inscrit en 1987 et étendu en 2002. Elle interroge l'utilisation de la reconnaissance de l'UNESCO dans la promotion touristique et, surtout, jauge la place que peut tenir le patrimoine non inscrit à proximité des sites inscrits. En effet, qu'advient-il des éléments qui sont exclus des périmètres d'inscription et qui, conséquemment, se retrouvent au ban des circuits touristiques? Si la Convention exprime l'importance de conserver aussi ce qui n'est pas inscrit, la réalité locale est parfois différente et il peut être difficile de sensibiliser les uns et les autres à la préservation de ce "patrimoine périphérique». Dans le cas de Budapest, il semble cependant que l'attention patrimoniale ne se soit pas limitée au périmètre de l'inscription, au bénéfice d'ailleurs de pratiques touristiques diverses.

Manon Istasse propose d'examiner à Fès, inscrite en 1981, diverses modalités de rencontre entre le tourisme et le patrimoine mondial. Trois cas de figure se présentent. Dans la médina d'abord, les propriétaires, principaux responsables de la préservation, sont surtout confrontés aux normes nationales et maîtrisent mal les enjeux et les bénéfices éventuels de l'inscription. Quant à elles, les autorités locales usent plutôt de l'inscription au titre d'instrument de promotion. Enfin, au sein de l'UNESCO, tant à Rabat qu'à Paris, on découvre un discours sur la gestion conjointe et durable entre le patrimoine mondial et le tourisme. Istasse souligne la complexité de cette relation polymorphe et propose de la considérer comme un "référent circulant» qui changerait de forme selon les situations tout en restant le même dans son essence.

Wided Majdoub analyse les relations entre les parties prenantes de la gestion de la médina de Sousse, inscrite en 1988. Après une évaluation des possibles raisons du manque d'intérêt pour la médina, à laquelle les touristes semblent préférer les attributs balnéaires de la ville, Majdoub tente de comprendre les dynamiques des acteurs, dont les animosités professionnelles grèvent l'essor de l'activité touristique dans la médina et, à terme, émargent au développement local.

Les articles de ce dossier invitent donc à questionner non seulement les fondements du tourisme et ses relations avec le patrimoine, plus particulièrement mondial, mais aussi les modulations particulières que ces éventuelles relations imposent sur les scènes locales, dans des contextes et des milieux fort différents. En deçà de la vision globale à laquelle invitent presque spontanément la reconnaissance de l'UNESCO et les qualificatifs de mondialité et d'universalité qu'elle convoque, comprendre la relation mondialisée entre patrimoine et tourisme requiert d'abord un examen local des déclinaisons politiques, sociales, culturelles et économiques du patrimoine mondial. En cette année qui marque le $40^{\mathrm{e}}$ anniversaire de la Convention concernant la protection du patrimoine mondial, culturel et naturel, la recherche universitaire a encore fort à faire pour cerner les enjeux et les aboutissants de cette patrimonialisation et de cette mise en tourisme spécifiques, eu égard aux habitants, aux pratiques sociales et culturelles, à l'environnement urbain ou naturel, sans compter les impacts sur le foncier, qu'on ignore trop souvent et qui pourtant, ostensiblement, reste le bien par essence du patrimoine.

Souhaitons que ce dossier de Téoros pave la voie des débats et des discussions sur le patrimoine mondial, appelés par les situations qu'on y explore sur la place du patrimoine dans le tourisme (et l'inverse), sur la gestion durable de ces deux phénomènes et sur l'avenir, au-delà du millier de sites, du patrimoine mondial.

Note

1 Selon l'édition de janvier 2012 du baromètre de l'Organisation mondiale du tourisme (http://mkt.unwto.org/fr/ barometer). 